\title{
El papel de las organizaciones empresariales y sindicales en la política social de atención a la dependencia.
}

\section{Vicente Marbán Gallego}

Dpto. de Fundamentos de Economía, Historia Económica y Sociología.

Universidad de Alcalá

<vicente.marban@uah.es>

Espainiako gizarte-politikaren baitan, mendetasunegoeran dauden pertsonen zaintzarako egungo erreformak bideratu izandako kultura, gizarte eta instituzioen aldaketaren analisia burutzen da, eta horren ostean, gizarte-eragileen (sindikatu eta enpresarioak) rolak aztertzen dira berauen egitekoa ezagutzearren. Partikularki, erantzuna eman nahi izan zaie honako galdera hauei: zein izan da gizarte-eragileen rolaren eragina egungo mendetasun-egoerako pertsonen artatzerako gizarte-politikaren itxuratzean, eta, modu berezian, Mendetasunaren Legean?; edota zein estrategia edo eragiteko gaitasun erabili dituzte erreforma hori burutzeko? Analisia osatzeko, berriz ustiatu dira Mendetasun Legea lantzeko partaide izandako zenbait gizarte-eragile estrategikori modu sakonean eginiko hamazazpi elkarrizketa. Besteak beste, galdegindako hauek hartu dituzte kontuan: sindikatuak eta enpresaburuak, irabazi-asmo gabeko erakundeak, alderdi politikoak, autonomiaerkidegoak eta adituak.

\section{HITZ-GAKOAK:}

Mendetasun Legea, epe luzeko zaintza, enpresaburuen elkarte, sindikatuak, hirugarren sektorea.
En este texto se parte del análisis de los cambios culturales, sociales e institucionales que han propiciado la actual reforma de la política social de atención a la dependencia en España para, a continuación, centrarse en el papel que los agentes sociales (sindicatos y empresarios) han desempeñado en dicha reforma. En particular, se pretende responder a preguntas como ¿cuál ha sido el papel de los agentes sociales en la actual configuración de la política social de atención a la dependencia y, en concreto, en la Ley de Dependencia?, 0 ¿cuáles han sido sus estrategias y capacidades de acción en dicha reforma? Este análisis se ha basado en una reexplotación de diecisiete entrevistas en profundidad realizadas a actores sociales estratégicos en la elaboración de la Ley de Dependencia, como los propios sindicatos y la patronal, así como entidades no lucrativas, partidos políticos, comunidades autónomas y expertos.

\section{Palabras Clave:}

Ley de Dependencia, cuidados de larga duración, organizaciones empresariales, sindicatos, tercer sector.

${ }^{1}$ Una versión preliminar de este artículo se presentó en el III Congreso Anual de la Red Española de Política Social, celebrado del 24 al 26 de noviembre en Pamplona. Esta versión ha sido mejorada gracias a las aportaciones de los asistentes al panel en el que se presentó la ponencia. 


\section{Introducción}

El 1 de enero de 2007 comenzó a aplicarse en España la Ley 39/2006, de Promoción de la Autonomía Personal y Atención a las Personas en Situación de Dependencia (LAPAD), conocida como Ley de la Dependencia, que crea un nuevo sistema de protección social, conocido como Sistema de Autonomía y Atención a la Dependencia (SAAD). Esta reforma es fruto de un conjunto de cambios institucionales, sociológicos e ideológicos que coinciden progresivamente a lo largo de casi quince años (entre 1992 y 2006), y actúa de manera desigual y con distinta intensidad (Rodríguez Cabrero, 2009; Rodríguez Cabrero y Marbán, en prensa).

Se trata de una reforma compleja, que pretende dar respuesta al mismo tiempo a varios objetivos:

- Asistenciales: cubrir un riesgo tradicional, garantizando un mínimo de protección en todo el territorio del Estado; apoyar a la familia cuidadora mediante prestaciones sociales, a la vez que facilitar la incorporación de la mujer al mercado de trabajo; mejorar la calidad de los servicios; promover la autonomía personal; o crear una red pública de servicios sociales de gestión mixta en todo el Estado.

- Institucionales: impulsar la cooperación institucional entre administraciones; promover la participación de los agentes sociales y económicos en el SAAD; garantizar las condiciones de igualdad en todo el territorio; o conciliar la vida familiar, los cuidados informales y el empleo.

- Económicos: crear empleo en servicios sociales, reforzar la cooperación financiera entre administraciones, impulsar la participación de los beneficiarios en la financiación (copago).

Esta multiplicidad de objetivos ha producido no escasos conflictos institucionales y financieros (entre administraciones), entre prestaciones y servicios (voto mayoritario a favor de las prestaciones económicas frente a los servicios) y entre los proveedores privados y el Estado (por el coste de los servicios y el escaso impulso al desarrollo de los servicios sociales). También ha producido una confluencia inercial de intereses entre los distintos actores sociales implicados (comunidades autónomas, corporaciones locales, partidos políticos, organizaciones del tercer sector del ámbito de los mayores y las personas con discapacidad, y sindicatos y organizaciones empresariales).

En este texto analizaremos tanto los factores culturales, sociales e institucionales que subyacen a la política social de atención a la dependencia, como el proceso de negociación y consenso entre los agentes sociales implicados. Para este análisis, nos hemos apoyado en una reexplotación de los resultados de diecisiete entrevistas en profundidad realizadas a agentes sociales y actores sociales estratégicos (entidades no lucrativas de acción social, partidos políticos, comunidades autónomas y expertos) entre octubre de 2007 y julio de 2008 , centrándonos en el análisis específico que estos actores hacen del papel desempeñado por los sindicatos y la patronal en el desarrollo de la LAPAD.

\section{Factores de cambio subyacentes a la política social de atención a la dependencia}

En la puesta en marcha del Sistema Español de Atención a la Dependencia, se entrecruzan varios conjuntos de intereses que han dado lugar a una ley compleja, basada en la necesaria cooperación institucional o administrativa entre el Estado y las comunidades autónomas. Detrás de esta rama de protección social, subyacen distintas cuestiones: el proceso de europeización cognitiva de las políticas sociales; la necesaria racionalización de los programas de atención existentes en la Seguridad Social, las comunidades autónomas y Ayuntamientos; la creciente demanda social de las familias; o la importancia del diálogo social, a través del cual se han canalizado las demandas de los sindicatos (en pro de nuevos nichos de empleo) y de los empresarios (en pro de nuevos ámbitos de gestión privada de los servicios sociales).

El principal episodio de la reforma de la política social de atención a la dependencia es la Ley 39/2006, de Promoción de la Autonomía Personal y Atención a las Personas en Situación de Dependencia. Su existencia y configuración no pueden explicarse sin atender al contexto cultural, social e institucional, los cuales, a su vez, han ejercido gran influencia en la postura de los diferentes actores sociales respecto el modelo español de atención a las personas dependientes, así como a las coaliciones entre dichos agentes.

\subsection{El contexto europeo y las inercias socioculturales e institucionales en el ámbito sociosanitario}

Un factor de cambio fundamental en la definición del actual sistema de atención a la dependencia español son las reformas políticas y las inercias institucionales, tanto internas como externas, en el ámbito sociosanitario.

En el plano externo, no podemos obviar el 'efecto llamada' que han tenido las reformas europeas en el debate sobre la atención a la dependencia en España. En particular, la incardinación del debate español en las políticas de la UE sobre esta materia ha supuesto la dinamización de un debate incipiente desde la década de 1990 (Marbán, 2011b). En la Unión Europea, la extensión y diversificación de los cuidados de atención a la dependencia se produjeron a distintas velocidades. A principios de los ochenta, los países nórdicos ampliaron y diversificaron su sistema de cuidados en respuesta a unos cambios sociodemográficos precoces en el contexto europeo (en especial, en Suecia, donde en el año 1980, 23 de cada 100 ciudadanos eran mayores de 65 años). 
Tales cambios todavía no se habían manifestado con una acusada intensidad en los países con sistemas contributivos de Seguridad Social hasta entrados los noventa. Los países nórdicos optaron, a principios de los ochenta, por integrar progresivamente las necesidades de atención a la dependencia en el extenso sistema de prestaciones y servicios sociosanitarios existentes. Una década después, mientras en los países nórdicos el debate latente sobre el sistema giraba en torno a su redimensionamiento, en el Reino Unido se discutía en torno a la búsqueda de soluciones para resolver el conflicto existente entre un sistema sanitario universal y unos servicios sociales asistenciales de atención a la dependencia.

En los sistemas de seguros sociales continentales, empezaron a gestionarse sistemas de seguros públicos para la cuidados de larga duración (Austria en 1993, Alemania en 1995, Luxemburgo en 1998 , Flandes en 2001, o Francia en 2002), en respuesta, además de a factores sociodemográficos, a un sistema que contaba con un alto nivel de copago -sobre todo en cuidados institucionales-, baja cobertura de prestaciones, disminución en la oferta de cuidadores familiares, y servicios de atención muy medicalizados y costosos (Pacolet, 1998).

Debe apuntarse, por lo tanto, cómo las experiencias europeas, en particular las de los países nórdicos y centroeuropeos (Pacolet, 1998, 2006), supusieron un acicate más en la definición de una política integral de atención a la dependencia en España (Pavolini y Ranci, 2008). Por ejemplo, tales experiencias se reflejan desde 2007 en los informes conjuntos de la Comisión Europea (2009, 2007a, 2007b, 2007c).

En el plano interno, nuestra reforma de la política social de atención a la dependencia también se construye a partir de un sistema de servicios sociales que ha ido aumentando considerablemente las ratios de cobertura en los últimos años, y también a partir de los siguientes hitos y reformas políticas de calado en la actual configuración de la política de atención a la dependencia (Rodríguez Cabrero, 2004; Rodríguez Rodríguez, 2006):

- La Constitución Española de 1978, en particular, sus artículos 49 y 50, que se refieren a la protección, por parte de los poderes públicos, de las situaciones de discapacidad y de las personas mayores, respectivamente.

- La Ley 13/1982, de 7 de abril, de Integración Social de los Minusválidos (LISMI). En virtud del artículo 49 de la Constitución, la LISMI contemplaba (artículo 16) un subsidio de ayuda por tercera persona, que fue suprimido en 1994, y al cual podían acceder quienes tuvieran 18 o más años, y no desarrollaran actividad laboral, tuvieran un grado de discapacidad igual o superior al $75 \%$ y la necesidad de asistencia de otra persona para realizar los actos más esenciales de la vida.

- El Plan Concertado para el Desarrollo de Prestaciones Básicas de Servicios Sociales de las Comu- nidades Locales, aprobado en 1988. En 1993, la Federación Española de Municipios y Provincias (FEMP) aprobó un catálogo que atribuía mayores competencias a las corporaciones locales en materia de servicios sociales, lo que implicó la extensión de servicios sociales como la ayuda a domicilio, una apuesta de los ayuntamientos hacia los cuidados de proximidad, y una incipiente dotación de profesionales municipales.

- La aprobación del Plan Gerontológico en 1992 supuso que, por primera vez, se abordaran de manera integral toda una serie de políticas en materia de envejecimiento, más allá de sus efectos sobre las pensiones, abriéndose camino la importancia de su atención sociosanitaria. Por entonces, el plan ya contemplaba medidas como alcanzar ratios suficientes en los principales servicios de atención, un complemento de pensión para pensionistas en situación de dependencia funcional, o mapas sociosanitarios y unidades específicas para coordinar los servicios sociales y sanitarios. Sin embargo, en su mayoría estas medidas no se implementaron por la falta de financiación e impulso político y por la fragmentación de competencias entre las administraciones públicas (Rodríguez Rodríguez, 2006). De haberse llevado a cabo, habrían supuesto un importante avance en la atención de situaciones de dependencia de personas mayores (Sancho y Rodríguez, 2002).

- Ley General de la Seguridad Social (texto refundido de 1994). El artículo 136, capítulo V del título II regula la incapacidad permanente, entre las cuales está la gran invalidez, definida como "la situación del trabajador afecto de incapacidad permanente y que, por consecuencia de pérdidas anatómicas o funcionales, necesita la asistencia de otra persona para los actos más esenciales de la vida, tales como vestirse, desplazarse, comer o análogos”. Asimismo, el artículo 181.a reconoce una asignación económica por cada hijo menor de 18 años a cargo del beneficiario, ayuda que también pueden cobrar las personas mayores de edad afectadas por una discapacidad en un grado igual o superior al $65 \%$.

- Las leyes autonómicas de servicios sociales -ya sean de primera generación (reguladas durante la década de 1980 y parte de la de 1990) como de segunda- en comunidades autónomas como Galicia, País Vasco, Comunidad de Valencia, La Rioja, Principado de Asturias, Madrid y Murcia (Guillén, 2005; Guillén y Vilá, 2007) han ido configurando, junto con diversos programas sociosanitarios y planes de atención a personas mayores, un mapa autonómico de servicios sociales cada vez más extenso. Aunque "se proclaman como universales, es decir, accesibles a cualquier persona en situación de necesidad [...], se trata de un derecho puramente retórico, que no puede compararse de ninguna manera al derecho subjetivo que tienen los sistemas de protección ya consolidados" (Rodríguez Rodríguez, 2006: 50). 
- Son igualmente reseñables otras medidas contempladas desde el ámbito sanitario, como la Ley General de Sanidad (1986), la práctica universalización de la atención sanitaria, y la Ley 16/2003, de Cohesión y Calidad del Sistema Nacional de Salud. Esta última regula, por primera vez, la prestación llamada sociosanitaria, que, si bien no se remite exclusivamente a la dependencia, es relevante para ella, pues incluye prestaciones de cuidados de larga duración, atención sanitaria durante la convalecencia y la rehabilitación en pacientes con déficit funcional recuperable. Según su artículo 14, tales prestaciones estarían destinadas a "aquellos enfermos, generalmente crónicos, que, por sus especiales características, puedan beneficiarse de la actuación simultánea y sinérgica de los servicios sanitarios y sociales para aumentar su autonomía, paliar sus limitaciones o sufrimientos, y facilitar su reinserción social".

- Finalmente, se deben mencionar otros hitos, como la creación, en 1988, del Ministerio de Asuntos Sociales, para tratar los servicios sociales de manera diferenciada a otros ministerios, y que supuso un cambio simbólico y representativo del creciente peso asignado a este pilar del Estado del bienestar; o la entrada en la agenda internacional con la celebración en España de la II Asamblea Mundial de Envejecimiento de Naciones Unidas (2002).

\subsection{Cambio cultural en torno a la responsabilidad de los cuidados}

Un segundo factor que hay que considerar es el cambio cultural producido en torno a la responsabilidad de los cuidados. Se trata de una transformación progresiva, que no implica un retroceso del modelo de atención familiar de cuidados, sino una profunda restructuración social interna.

Ese cambio cultural viene desencadenado, en primer lugar, por procesos sociodemográficos que afectan a la dinámica y los roles familiares tradicionales, como el menor tamaño de las familias o la creciente participación de la mujer en el mercado laboral. Tales cambios están precipitando una crisis de la red de cuidados de la que disponían las personas dependientes en décadas anteriores, $y$ han puesto de manifiesto la fragilidad e insuficiencia de un modelo de atención como el actual, sustentado en las mujeres cuidadoras: en el $22,5 \%$ de los casos, el cuidador principal suele ser el cónyuge y, fundamentalmente, las hijas (39,3\% frente al 9,15\% de los hijos) [Centro de Investigaciones Sociológicas, 2006].

En cierto modo, como consecuencia de esas transformaciones sociodemográficas, se ha registrado una creciente demanda de socialización de los cuidados, es decir, de asunción de responsabilidades colectivas en los cuidados. Así, en 2004, el 10,6\% de los mayores de 65 años creían que las familias debían asumir toda la responsabilidad de los cuidados, mientras que dos años después, esa opinión la sostenía el $5,5 \%$. Del mismo modo, el porcentaje de personas que opinaba que la familia debía ser responsable de los cuidados con ayuda de las administraciones públicas pasó del 38\% en 2004 al 43\% en 2006 (Centro de Investigaciones Sociológicas, 2006; Imserso, 2004).

Parte de este cambio en la cultura de los cuidados viene también desencadenado por un factor menos visible, pero que ha operado de manera latente y gota a gota en el imaginario colectivo: la creciente densidad de conciencia con respecto a la dependencia. Dicho factor parte, en los años ochenta, de un movimiento ilustrado encabezado por las clases profesionales provenientes del ámbito de la Seguridad Social, y de la geriatría y la gerontología, así como de expertos y académicos que trasladan esas inquietudes a las administraciones públicas, las cuales reaccionan, no obstante, con retraso a las demandas sociales planteadas. Se trataba de profesionales y expertos que, en muchos casos, conocían las experiencias de otros países en el campo de la dependencia, la geriatría y la gerontología. Contribuyeron a crear incipientes cambios en la cultura sobre la responsabilidad de los cuidados, una especie de 'conciencia por goteo' sobre dicho fenómeno, y sus conocimientos y experiencias posteriormente se pusieron relieve en el libro blanco (Rodríguez Castedo, 2005).

Dicho 'movimiento ilustrado' consiguió que, desde finales de los ochenta y principios de los noventa, la dependencia se incorporarse a la 'agenda de asuntos pendientes' de nuestro Estado del bienestar, hecho en cierto modo también propiciado porque, en esos años, el principal debate sobre protección social girara en torno a la viabilidad del sistema de pensiones y sus posibles reformas. No sería hasta iniciada la primera década de 2000 cuando la demanda social creciente de un buen número de familias 'desbordadas' por un aumento de la esperanza de vida con elevados años de discapacidad y plenamente concienciadas del problema fuera canalizada por los sindicatos y las organizaciones sociales de la discapacidad y de personas mayores, a través de consejos estatales y plataformas de entidades (Plataforma de ONG de Acción Social, Consejo Estatal de Personas Mayores, Consejo de ONG de Acción Social, Comité Español de Representantes de Personas con Discapacidad).

\subsection{La provisión de cuidados a la dependencia}

En tercer lugar, la reforma de la política social de atención a la dependencia no puede explicarse sin atender a la organización y la insuficiente provisión de los cuidados en las esferas pública y privada para sostener o impulsar un sistema de atención a la dependencia de amplia cobertura, como se pretende en nuestro recién estrenado sistema. 
En este sentido, el caso español participa de algunos de los rasgos comentados a escala de la UE: alta feminización de los cuidados y significativa prevalencia de los cuidados informales; creciente colaboración público-privada en la provisión de los servicios, con un peso creciente de la iniciativa privada lucrativa y no lucrativa; gestión descentralizada de la provisión de servicios; creciente regulación de la provisión privada de los servicios; y (re)definición de los estándares de evaluación, seguimiento y control de la calidad de las prestaciones.

Estos rasgos son consustanciales a nuestro sistema de cuidados, de trayectoria asistencial y sustentado por las familias, las entidades no lucrativas, las empresas privadas (fundamentalmente en el ámbito de los servicios residenciales, donde el índice de cobertura de las residencias privadas es del 2,28, superior al de las residencias públicas $(2,04), y$ donde el $80 \%$ de las residencias son de titularidad privada (Imserso, 2008) y con una participación multinivel de perfil asistencialista de las administraciones públicas, en especial las comunidades autónomas y las corporaciones locales.

Efectivamente, se constata cómo las familias son las primeras proveedoras de cuidados y cómo recaen sobre ellas de manera muy significativa los principales cuidados de las personas mayores. Así se ponía de manifiesto en la Encuesta de condiciones de vida de los mayores 2006 (Centro de Investigaciones Sociológicas, 2006), ya que, mientras que los cuidadores principales familiares (cónyuge, hijas e hijos) se ocupaban, por término medio, del $71 \%$ de los cuidados principales, los servicios sociales públicos o las empresas de servicios sociales privadas tan sólo lo hacían en el 2,5\% y el 0,2\% de los casos, respectivamente.

En cuanto al papel de las administraciones públicas, existe un amplio mapa de prestaciones y ventajas fiscales para atender a la dependencia. Se trata de prestaciones que, si bien han aumentado significativamente en oferta y cobertura en los últimos años, son insuficientes y están desequilibradas territorialmente en tasas de cobertura, requisitos de acceso y en aportaciones del usuario (Marbán, 2009).

En definitiva, con la organización y provisión de los cuidados previa a la LAPAD, los servicios y las prestaciones seguían siendo insuficientes, y la sobrecarga de los cuidadores familiares, excesiva, lo cual, junto a la inmediata demanda de necesidades de atención, justifica plenamente la puesta en marcha de la reforma y responde a nuestra pregunta de por qué era necesaria una política integral de atención a la dependencia en España como la implementada hace cinco años con la LAPAD.
2.4. La atención a la dependencia: una reforma gradual, pero con interrupciones

Como ya hemos puesto de manifiesto (Marbán, 2011a), este recorrido por los factores de cambio subyacentes a la política social de atención a la dependencia apunta a que la LAPAD no constituye un episodio de cambio o una reforma radical, sido una reforma gradual, dependiente de una senda (path dependency) acumulativa de factores socioeconómicos e institucionales acaecidos desde los años ochenta, que amplía y complementa la acción protectora que ya venía realizando el Estado y el sistema de Seguridad Social.

No obstante, en esa senda acumulativa de factores, la política social de atención a la dependencia también ha estado sometida, hasta iniciado el siglo XXI, a una sucesión de procesos interrumpidos, debido a que los sucesivos Gobiernos, y también otros agentes sociales, han ido posponiendo la atención a la dependencia como una prioridad en ciernes. Podríamos apuntar varias circunstancias que avalarían esta fundamentación.

En primer lugar, conviene recordar de nuevo cómo el Plan Gerontológico, de haberse implementado plenamente desde 1992, habría supuesto un importante avance en la atención a situaciones de dependencia de personas mayores, que se vio, en cierto modo, 'interrumpido' por la falta de financiación e impulso político y por la fragmentación de competencias entre las administraciones públicas.

Igualmente, sucede que durante el segundo mandato del Partido Popular, entre 2000 y 2004, tanto la creación de una Comisión sobre Dependencia -denominada también Comisión Zaplana- como el Acuerdo Social de 2001 y la situación económica de elevado crecimiento económico del momento indujeron a pensar que la Ley de la Dependencia la haría el Partido Popular en ese mandato. Sin embargo, eso no sucedió, probablemente por razones presupuestarias, como apunta en su entrevista uno de nuestros informantes.

En tercer lugar, en lo que respecta a los sindicatos, la dependencia tardó en entrar en su agenda, en la medida en la que se priorizaron aspectos como la dignificación de las pensiones o la protección al desempleo (debido, en buena medida, a las altas tasas de paro de la década de 1990). En cuanto a las organizaciones empresariales, no participaron de esta preocupación -más allá de su posible efecto en los seguros privados de cuidados de larga duraciónhasta que no empezaron a constatar el posible espacio de negocio de la dependencia -especialmente en la atención a personas mayores-y el clima favorable hacia la protección social de esta contingencia. 


\section{El papel de los sindicatos y las organizaciones empresariales en la reforma de la política social de atención a la dependencia}

Además de por la acumulación de los factores mencionados, la LAPAD también es el resultado de la importancia central, que no determinante, de la confluencia inercial de intereses entre los distintos actores sociales implicados (comunidades autónomas, corporaciones locales, partidos políticos, organizaciones del tercer sector del ámbito de los mayores y las personas con discapacidad, y sindicatos y organizaciones empresariales).

En síntesis, la gestación de la Ley de Dependencia arrancó con la elaboración del Libro blanco de la dependencia (Rodríguez Castedo, 2005) en mayo de 2004, por iniciativa del Gobierno del PSOE, a través del Imserso, y se publicó a principios de enero de 2005. Sobre la base de dicho documento, el contenido de la futura ley fue debatido desde inicios de ese año por patronal, sindicatos y Gobierno. En diciembre de 2005, se alcanzó un acuerdo social entre el Gobierno, los sindicatos UGT y CC.00., y las patronales CEOE y CEPYME, mediante el cual se acordaron los principios básicos del modelo (financiación, prevalencia de servicios sobre prestaciones, desvinculación de la Seguridad Social y sistema universal).

¿Cuál ha sido el papel de los agentes sociales en la actual configuración de la política social de atención a la dependencia y, en particular, en la Ley de Dependencia? ¿Cuáles han sido sus estrategias y capacidades de acción en dicha reforma? La respuesta a estas cuestiones requiere diferenciar dos escenarios:
- Un escenario de consenso (Figura 1) articulado en torno a una serie de aspectos básicos de la ley sobre los cuales existió un relativo acuerdo -aunque por razones e intereses distintos- entre los actores sociales, como la universalidad y la igualdad de acceso, el reconocimiento de los derechos de los cuidadores en la Seguridad Social, la importancia concedida a la prevención, la formación, la calidad en la prestación de servicios o la importancia del apoyo a las familias.

- En lo relativo a la universalidad y la igualdad de acceso al sistema, los sindicatos perseguían la consolidación de la oferta pública de servicios; y la patronal, la consolidación de la oferta privada. En el caso de las organizaciones empresariales, aunque cabía esperar que abogaran por un seguro privado de dependencia, finalmente optaron por un sistema universal, no sólo por el alto consenso sociopolítico en torno a dicho sistema, sino sobre todo por la necesidad de contar con un marco jurídico estable que garantizara la provisión mixta de los servicios de atención a la dependencia y, por ende, la posibilidad de dar salida a sus excedentes de plazas residenciales.

- Los agentes sociales también coincidían en la preferencia por un modelo de prestaciones en servicios sobre un modelo de prestaciones monetarias. Con ello, los sindicatos pretendían potenciar la creación de empleo y la integración laboral femenina, al objeto de que hubiera servicios de respiro suficientes que facilitan el tránsito de las mujeres cuidadoras al mercado laboral. Las organizaciones empresariales querían consolidar la demanda de servicios, la gestión privada de los servicios sociales, la capacidad instalada en servicios residenciales y de ayuda a domi-

Figura 1. Aspectos básicos de consenso entre los agentes sociales respecto a la Ley de Dependencia

Gestión mixta

de la red de

servicios sociales

$\uparrow$

Sindicatos: creación de empleo de calidad, integración laboral femenina.

Patronal: consolidación de la capacidad instalada en servicios residenciales y ayuda a domicilio, absorción de excedentes de plazas residenciales.

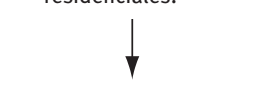

Prevalencia de servicios sobre prestaciones económicas
Sindicatos: consolidación de la oferta pública de servicios, gestión pública de los servicios sociales de responsabilidad pública.

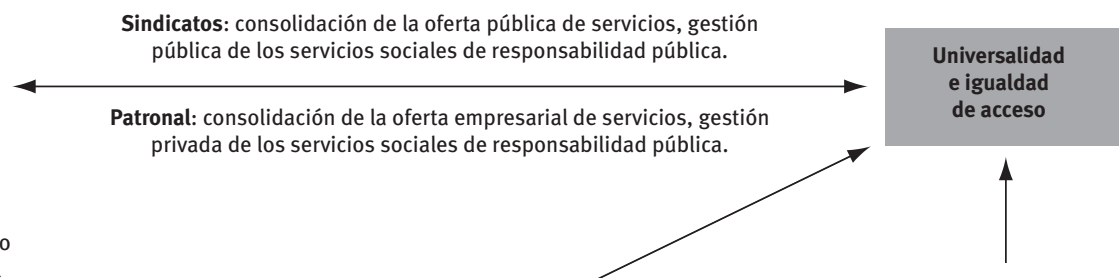

Sindicatos: creación de empleo de calidad, integración laboral femenina. Patronal: consolidación de la capacidad instalada en servicios residenciales y de ayuda a domicilio, absorción de excedentes de plazas residenciales.
Sindicatos: oferta de formación. Patronal: mejora de la calidad del servicio.

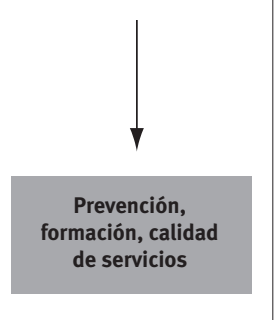

Fuente: Elaboración propia. 


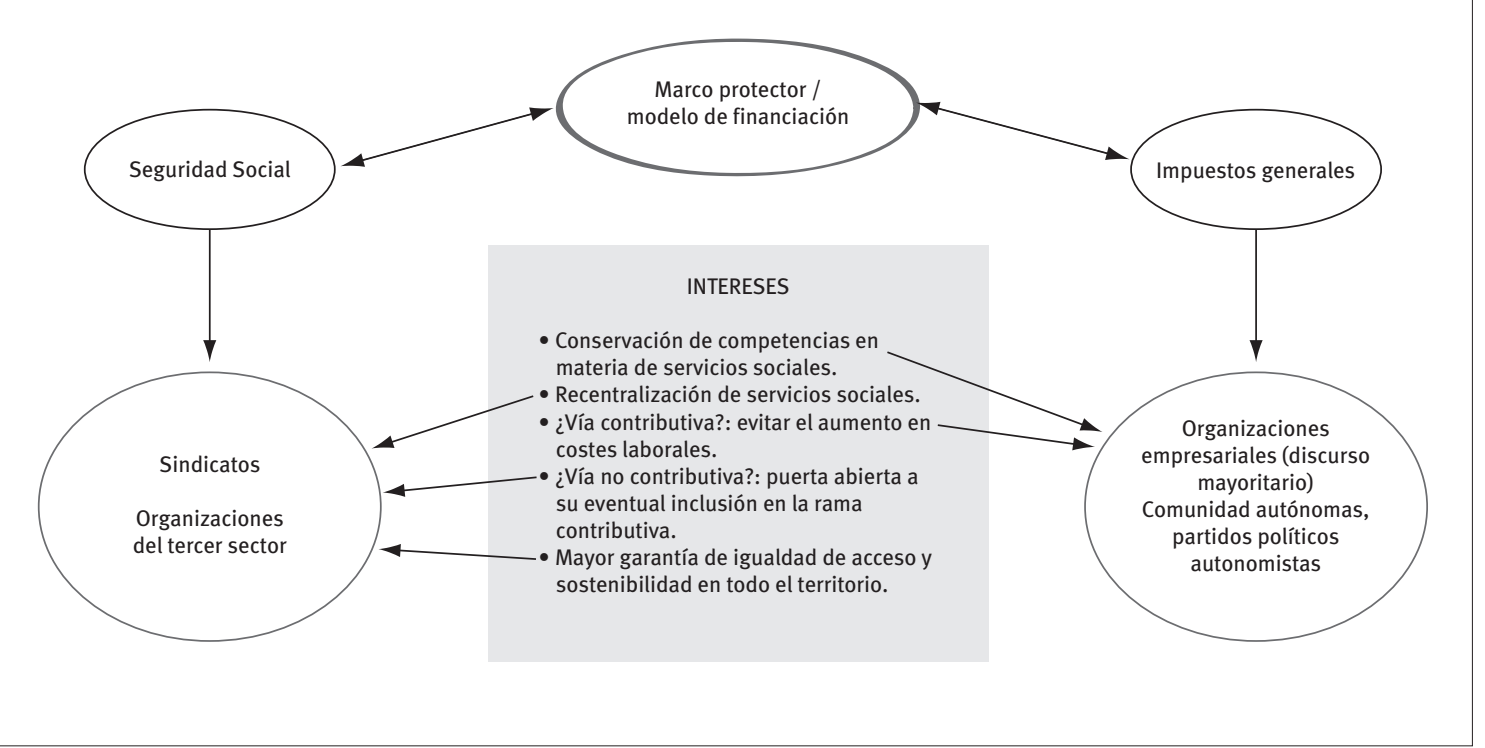

Fuente: Elaboración propia.

cilio, y la absorción de excedentes de plazas residenciales.

- Un escenario de disenso (Figura 2), en torno a aspectos como el marco protector y el ámbito competencial, que fueron objeto de intenso debate.

Con respecto al marco protector y el ámbito competencial, el principal debate surgió en torno a dos cuestiones: a) si la LAPAD debía incluirse en el marco de la Seguridad Social, o bien dejarse fuera de ella; y b) la consiguiente financiación vía impuestos. La primera posibilidad podría suponer un aumento en las cotizaciones (en el caso de ir por la vía contributiva), así como la transferencia de más competencias en servicios sociales a la administración central, en detrimento de las comunidades autónomas.

A este respecto, la patronal, gran parte de las comunidades autónomas y los partidos políticos de corte más autonomista se oponían a la inclusión de la Ley de Dependencia en el marco de la Seguridad Social y defendían la financiación vía impuestos:

- Para las organizaciones empresariales, enmarcar la LAPAD en la Seguridad Social por la vía contributiva implicaría un aumento en sus costes laborales (en cotizaciones sociales), lo cual lastraría su crecimiento organizativo, mientras que hacerlo por la vía no contributiva habría supuesto una puerta abierta a su inclusión en algún momento en la rama contributiva.

- Para la mayoría de las comunidades autónomas y los partidos políticos de corte más autonomista, incluir la LAPAD en el sistema de la Seguridad Social afectaba de pleno al ámbito competencial autonómico, pues conferiría al Gobierno central una legitimidad y una capacidad imperativa que invadía las competencias de las comunidades autónomas en el ámbito de servicios sociales, y les restaba legitimidad para intervenir en la práctica.

Ésa fue la posición prevalente de las organizaciones empresariales, lo cual no impidió que abogaran por un sistema de amplia cobertura que estableciera un marco estable de ordenamiento jurídico y de financiación para la atención a la dependencia. Más bien todo lo contrario: un marco jurídico estable y la definición de un sistema universal de amplia cobertura y de gestión mixta como el que se proponía en la LAPAD podría suponer, como hemos afirmado anteriormente, la consolidación de la capacidad instalada en servicios residenciales y de ayuda a domicilio, así como la absorción de excedentes de plazas residenciales del sector privado mercantil.

No obstante, hubo un debate interno en el seno de la CEOE -fruto de la desfragmentación del sector-, articulado en torno a la siguiente dualidad de discursos (Figura 3):

- Por un lado, el discurso sostenido por las grandes organizaciones empresariales y por el sector empresarial en general. Dicho discurso, mayoritario, considera la atención a la dependencia como un sector asistencial generador de gasto y donde el rechazo al modelo de Seguridad Social se fundamenta en la contención de costes laborales que supondría, desconsiderándose los efectos positivos de tal modelo, en términos de financiación estable y existencia de un marco regulador homogéneo, que evitaría incertidumbres en la acreditación de centros entre las diferentes comunidades autónomas. 


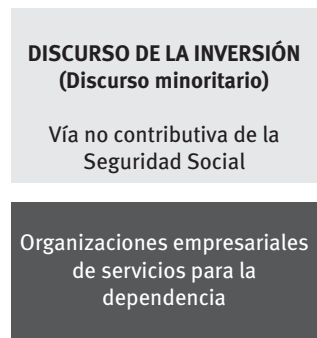

Sostenibilidad financiera

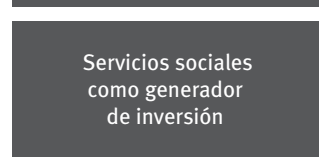

DISCURSO DEL GASTO

(Discurso mayoritario)

Desvinculación de la

Seguridad Social:

financiación vía impuestos

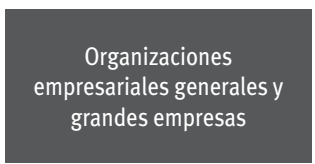

Contención de costes laborales

Servicios sociales

como sector asistencia

generador de gasto

Fuente: Elaboración propia a partir del análisis de las entrevistas.

El discurso sostenido por las organizaciones empresariales de servicios para la dependencia, que conciben la atención a esta contingencia como un espacio generador de inversiones y de creación de empleo no deslocalizable. En dicho discurso, prevalecen la seguridad jurídica y económica que les aportaría el marco regulador de la Seguridad Social, más estable y homogéneo, para evitar los elevados costes de transacción que, para estas organizaciones, tienen aspectos como la acreditación de centros.

Por el contrario, las organizaciones del tercer sector y los sindicatos eran proclives a incluir los derechos y las prestaciones en el ámbito de la Seguridad Social, y a una participación más activa de la administración central en los servicios sociales, fundamentalmente porque, con ellos, se respondía con mayor fidelidad a la igualdad de acceso en todo el territorio, a la extensión de los servicios públicos, o a la extensión de los servicios privados no lucrativos de responsabilidad pública.

La postura inicial de los sindicatos con respecto al marco protector de la LAPAD fue situar la dependencia dentro del ámbito de la Seguridad Social, aunque no necesariamente en la parte contributiva; de hecho, se contempló la posibilidad de incluir el sistema en la vía no contributiva de la Seguridad Social, pero gestionada por las comunidades autónomas. En este aspecto, fueron moderando sus posiciones hasta aceptar la vía impositiva, en especial por dos razones:

- Porque la integración de los mecanismos de protección de la dependencia en la Seguridad Social podría implicar un replanteamiento del Pacto de Toledo, que dejaba al margen del sistema contributivo todo lo que no fueran prestaciones econó- micas puras, teniendo en cuenta que el texto de la Ley de Dependencia prioriza las prestaciones en servicios sobre las económicas.

- Por la presión de las comunidades autónomas, las cuales "en el caso de que se incluyese dentro del ámbito de la Seguridad Social, iban a presentar un recurso de inconstitucionalidad" (UGT), lo cual podría retrasar en exceso la universalización de esta contingencia.

En definitiva, según las entrevistas realizadas, el hecho de no enmarcar el nuevo sistema en el ámbito de la Seguridad Social pudo deberse al rechazo de la patronal, los partidos políticos más autonomistas - los cuales poseían una mayor presencia parlamentaria durante la legislatura en que se aprobó la ley-, pero, sobre todo, a la oposición de las comunidades autónomas, respaldadas por su capacidad instalada en los servicios sociales y por las amenazas de inconstitucionalidad de algunas de ellas, que, de producirse, habrían podido retrasar la aprobación de una ley en un contexto socioeconómico propicio.

\section{Conclusiones}

La Ley de Dependencia representa un hito moderno en la configuración actual del Estado del bienestar. Su impacto, tanto en la economía (Montserrat, 2011; Sosvilla Rivero, 2010) como en el número de personas beneficiarias, está siendo cada vez más significativo. Como explica el recientemente publicado informe de evaluación de la Ley 39/2006 (Imserso, 2011), elaborado por un equipo de investigación independiente, a 1 de noviembre de 2011 hay más de 740.000 beneficiarios que reciben prestaciones, frente a un 
total de 914.373 que tienen reconocido ese derecho. En términos de empleo, los puestos de trabajo vinculados a la atención a la dependencia durante 2009 y 2010 han supuesto un total de 248.000 , y se han creado 165.000 nuevos empleos como resultado del nuevo gasto implementado con la LAPAD. El gasto sobre el PIB se ha doblado entre 2003 y el 2010, pasando del $0,32 \%$, a casi el $0,64 \%$, cifra que supone unos retornos fiscales de casi el $20 \%$.

Hemos puesto de manifiesto que, en su actual configuración, la LAPAD tiene su referente en una serie de factores culturales, sociodemográficos e institucionales que han ejercido gran influencia en el modelo vigente de atención a las personas dependientes. Entre otros, han resultado determinantes los siguientes factores: los cambios en la cultura sobre la responsabilidad de los cuidados, desencadenados por cambios sociodemográficos y una creciente demanda de socialización de esta contingencia; la insuficiente provisión de los cuidados en las esferas pública y privada, donde las familias son las primeras proveedoras de cuidados; así como las reformas políticas y las inercias institucionales tanto internas (reformas como el Plan Gerontológico o el Plan Concertado) como externas (europeización cognitiva de las políticas sociales).

Con ello, se evidencia que la LAPAD supone una reforma gradual dependiente de una senda acumulativa (path dependency) de factores socioeconómicos e institucionales acaecidos desde los años ochenta. En realidad, podría considerarse al nuevo sistema como el perfeccionamiento del sistema anterior, que se transforma en un sistema de cobertura universal, basado en un derecho subjetivo (como la sanidad o la educación) y en la cooperación entre el Estado y las comunidades autónomas o regiones, y financiado con impuestos, cotizaciones sociales y copago de los usuarios.

Por otro lado, la LAPAD también es el resultado de la confluencia inercial de intereses entre los distintos actores sociales implicados (comunidades autónomas, corporaciones locales, partidos políticos, organizaciones del tercer sector del ámbito de los mayores y las personas con discapacidad, y sindicatos y organizaciones empresariales). Aunque el debate comenzó antes, desde una perspectiva institucional el punto de partida fue el acuerdo social de diciembre de 2005 entre las organizaciones empresariales, los sindicatos y el Gobierno, en el que se acordaron los principios básicos del modelo (financiación, prevalencia de servicios sobre prestaciones, desvinculación de la Seguridad Social y universalidad del sistema).

Hubo cierto grado de acuerdo en torno a una serie de aspectos básicos de la ley, como la universalidad y la igualdad de acceso, la gestión mixta de la red de servicios sociales, el reconocimiento de los derechos de los cuidadores en la Seguridad Social, y la importancia concedida a la prevención, la formación o la calidad en la prestación de servicios. El consenso se produjo porque incorporar estos aspectos en la ley favorecía las expectativas de ambos agentes sociales de contar con un marco jurídico estable que garantizara la provisión mixta de los servicios de atención a la dependencia. Para los sindicatos, ello podría suponer consolidar la oferta pública de servicios y favorecer la creación de empleo y la integración laboral femenina. Para las organizaciones empresariales, representaba consolidar la oferta y la demanda de los servicios ofertados por las empresas del sector, y mayores posibilidades a la gestión privada de los servicios sociales de responsabilidad pública.

El principal punto de desacuerdo giró en torno al marco protector y el ámbito competencial, en particular, a la inclusión o no de la LAPAD en el marco de la Seguridad Social. Entre las organizaciones empresariales, prevaleció - no sin debate interno- la estrategia de rechazar la inclusión en la Seguridad Social tanto en la vía contributiva -por cuanto ello implicaría un aumento en sus costes laborales- como por la vía no contributiva -en la medida en que ello habría dotado a la Administración de mayor margen de maniobra para incluirla, en un futuro, en la rama contributiva-. En cuanto a las organizaciones sindicales, aunque su estrategia inicial fue la de apoyar la inclusión de la Ley de Dependencia en el marco de la Seguridad Social, finalmente aceptaron su financiación vía impuestos, no sólo por la negativa de las organizaciones empresariales al acuerdo social -con la connivencia del Ministerio de Economía y Hacienda-, sino sobre todo por la amenaza latente de que algunas comunidades autónomas plantearan un recurso de inconstitucionalidad, lo que, de haberse producido, habría podido retrasar en exceso la universalización de esta contingencia.

Con la perspectiva de los cinco años trascurridos desde la puesta en marcha de la LAPAD, cabría afirmar, no obstante, que el resultado final para las organizaciones empresariales no ha sido del todo satisfactorio en lo que se refiere a sus principales objetivos (Figura 4). Por un lado, han logrado excluir la ley del ámbito de la Seguridad Social (con el inestimable apoyo de las comunidades autónomas) y dar mayor visibilidad a ciertos productos de financiación privados (por ejemplo, la hipoteca inversa). Por otro, no han conseguido que el peso de la iniciativa privada en el conjunto de la ley sea especialmente significativo, sobre todo si lo comparamos con el asignado a las organizaciones del tercer sector, ni tampoco han podido dar salida a los excedentes de plazas en los servicios residenciales, debido, entre otras razones, a la prevalencia de las prestaciones económicas sobre las prestaciones en servicios.

En lo relativo a los sindicatos (Figura 4), aunque finalmente la LAPAD no se incluyera en el marco de la Seguridad Social, su papel fue significativo en la consecución de una ley de carácter universal que consolida un derecho subjetivo. Sucede, empero, que sus otros objetivos prioritarios -crear empleo, favorecer el trabajo femenino, reducir la precariedad laboral y mejorar la formación de los cuidadores profesiona- 


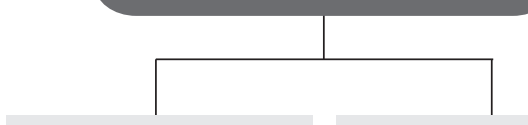

LAPAD fuera del ámbito de la Seguridad Social.

Mayor visibilidad a ciertos productos de financiación privados.
DEBILIDADES

Limitado reconocimiento de la iniciativa privada en el conjunto de la LAPAD.

Baja cobertura de excedentes de plazas en los servicios residenciales.

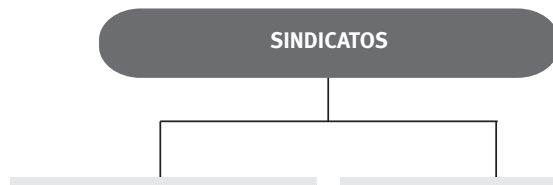

FORTALEZAS

Consolidación de un derecho subjetivo.

Reconocimiento de los derechos de los cuidadores.
DEBILIDADES

Resultados por debajo de expectativas en:

- Creación de empleo (330.000 para 2010, según el libro blanco).

- Precariedad laboral de los cuidadores profesionales

(en bajos salariosy temporalidad).

- Formación de cuidadores.

- Perpetuación de la mujer como cuidadora.

Fuente: Elaboración propia a partir del análisis de las entrevistas.

les- se han saldado, hasta la fecha, por debajo de las expectativas. En términos de empleo, los puestos de trabajo vinculados a la atención a la dependencia durante 2009 y 2010 han ascendido a 248.000 y se han creado 165.000 nuevos empleos como resultado del gasto nuevo implementado con la LAPAD (Imserso, 2011). Una cifra, que si bien queda por debajo de las previsiones de generación neta de puestos de trabajo del libro blanco -más de 330.000 en el horizonte de 2010 (Rodríguez Castedo, 2005)-, es de gran relevancia, habida cuenta de la importante destrucción de empleo en la mayoría de los sectores de la actividad.

La precariedad laboral de los cuidadores profesionales es todavía un hecho, debido a la existencia de unos márgenes muy ajustados en la concertación y los convenios con los centros residenciales y de ayuda a domicilio, lo que desemboca en bajos salarios y mayor temporalidad en relación a otros sectores. Del mismo modo, a pesar de los avances conseguidos, ya reflejados en distintos informes de evaluación de la LAPAD (Agencia Estatal de Evaluación de las Políticas Públicas y la Calidad de los Servicios, 2008; Barriga et al., 2009, 2010; Cervera et al., 2009; Imserso, 2011), las carencias existentes en la formación de cuidadores (Imserso, 2011), o la perpetuación de la mujer como cuidadora-debido a la significativa prevalencia de las prestaciones económicas- son todavía asignaturas pendientes. 
AGENCIA ESTATAL DE EVALUACIÓN DE LAS POLÍTICAS PÚBLICAS Y LA CALIDAD DE LOS SERVICIOS (2008): "La participación de la administración general del Estado en el Sistema para la Autonomía y Atención a la Dependencia”, Madrid, Agencia Estatal de Evaluación de las Políticas Públicas y la Calidad de los Servicios, [<http://www.aeval.es/comun/pdf/ evaluaciones/E13.pdf〉].

BARRIGA, L.; et al. (2010): Desarrollo e implantación territorial de la Ley de Promoción de la Autonomía Personal y Atención a las Personas en Situación de Dependencia. $V$ Dictamen, Asociación Estatal de Directoras y Gerentes en Servicios Sociales [<http:// www.directoressociales.com/documentos/ libroVdictamenjulio.pdf〉].

- (2009): Desarrollo e implantación territorial de la Ley de Promoción de la Autonomía Personal y Atención a las Personas en Situación de Dependencia. III Dictamen, Asociación Estatal de Directoras y Gerentes en Servicios Sociales [<http:// www.imsersomayores.csic.es/documentos/ documentos/barriga-dictamen-02.pdfs].

CENTRO DE INVESTIGACIONES SOCIOLÓGICAS (2006): Encuesta de condiciones de vida de los mayores, serie Estudios, no 2.647 [rhttp:// www.cis.es/cis/opencm/ES/1_encuestas/ estudios $/$ ver.jsp? estudio $=7740 \&$ cuestionario $=$ $8954 \&$ muestra $=14065$ >].

CERVERA, M. et al. (2009): Informe final del grupo de expertos para la evaluación del desarrollo y efectiva aplicación de la Ley 39/2006, de 14 de diciembre de Promoción de la Autonomía Personal y Atención a las Situaciones de Dependencia, Madrid, Imserso [<http://www. imserso.es/groups/imserso/documents/ binario/informesaadgexpertos.pdf $>$ ].
COMISIÓN EUROPEA (2009): Joint Report on Social Protection and Social Inclusion 2009, Luxemburgo, Oficina de Publicaciones de la Unión Europea [shttp://dx.doi. org/10.2767/51562>].

- (2007a): Comunicación de la Comisión al Consejo, al Parlamento Europeo, al Comité Económico y Social Europeo y al Comité de las Regiones. Propuesta de informe conjunto sobre protección social e inclusión social 2007 , Bruselas, Comisión Europea [rhttp://eur-lex. europa.eu/LexUriServ/site/es/com/2007/ com2007_0013es01.pdf〉].

- (2007b): Informe conjunto sobre protección social e inclusión social 2007. Perfiles de países, Bruselas, Comisión Europea.

- (2007c): Joint Report on Social Protection and Social Inclusion. Supporting Document, Bruselas, Comisión Europea [<http://ec.europa.eu/ employment_social/social_inclusion/ docs/2007/joint_report/sec_2007_329_ en.pdf>].

GUILLÉN, E. (comp.) [2005]: Sobre problemas y respuestas sociales, Barcelona, Hacer.

GUILLÉN, E.; y VILÀ, A. (2007): “Impacto de la Ley de Promoción de la Autonomía Personal y Atención a las Personas en Situación de Dependencia en los servicios sociales de las comunidades autónomas", en CASADO, D.; y FANTOVA, F. (coords.), Perfeccionamiento de los servicios sociales en España: informe con ocasión de la ley sobre autonomía y dependencia, Madrid, Fundación FOESSA; Cáritas, págs. 177-208.

IMSERSO (2011): Informe del Gobierno para la evaluación de la Ley 39/2006, de 14 de diciembre, de Promoción de la Autonomía Personal y Atención 
a las Personas en Situación de Dependencia (2007-2010), Madrid, Ministerio de Sanidad, Política Social e Igualdad [<http://www. dependencia.imserso.es/InterPresent1/ groups/imserso/documents/binario/ie_2011_ informeevaluacion.pdf $>$ ].

- (2008): Las personas mayores en España. Informe 2008, Madrid, Ministerio de Trabajo y Asuntos Sociales [rhttp://www.imsersomayores.csic. es/estadisticas/informemayores/informe2008/ index.html>].

- (2004): Encuesta de condiciones de vida de los mayores, Madrid, Imserso [<http://www. imsersomayores.csic.es/estadisticas/ encuestas/index.html>].

Ley 39/2006, de 14 de diciembre, de Promoción de la Autonomía Personal y Atención a las Personas en Situación de Dependencia [<http://www. boe.es/boe/dias/2006/12/15/pdfs/A4414244156.pdf >].

MARBÁN, V. (2011a): “Actores sociales y desarrollo de la Ley de Dependencia en España”, Revista Internacional de Sociología [<http:// revintsociologia.revistas.csic.es/index.php/ revintsociologia/article/view/428/451>].

- (2011b): "Los actores sociales en el sistema de dependencia español", Cuadernos de Relaciones Laborales, vol. 29, $\mathrm{n}-1$ 1, págs. 69 91 [hhttp://revistas.ucm.es/index.php/CRLA/ article/view/36186/35052〉].

- (2009): "La atención a la dependencia”, en MORENO, L. (coord.), Reformas de las políticas del bienestar en España, Madrid, Siglo XXI, págs. 207-238.

MONTSERRAT, J. (2011): Evaluación económica del SAAD, Barcelona, Mimeo.

PACOLET, J. (2006): “Protección social de las personas mayores dependientes: sostenibilidad de Estado del bienestar y ámbito del seguro de cuidados de larga duración", Revista Española del Tercer Sector, no 3, págs. 111-160 [<http://www.fundacionluisvives.org/rets/3/ articulos/6368/index.html s].

- (1998): Social Protection for Dependency in Old Age in the 15 EU Member Status and Norway, Bruselas, Comisión Europea.

PAVOLINI, E.; y RANCI, C. (2008): "Restructuring the Welfare State: Reforms in long-term care in Western
European countries", Journal of European Social Policy, vol. 18, no -3 , págs. 246-259 [<http://rszarf.ips.uw.edu.pl/welfare-state/ ranci.pdf $\rangle]$

RODRÍGUEZ CABRERO, G. (2009): "El desarrollo de la política social de promoción de la autonomía y atención a las personas en situación de dependencia en España (2007-2009)", Gestión y Análisis de Políticas Públicas, nํ2, págs. 33-58.

- (2004): "Protección social de la dependencia en España", serie Documentos de Trabajo, no 44, Madrid, Fundación Alternativas [<http://www.falternativas.org/content/ download/5708/163686/version/1/ file/431d_12-09-05_44_2004.pdf)].

RODRÍGUEZ CABRERO, G.; y MARBÁN, V. (en prensa): “Longterm care in Spain: A hybrid system of informal family care tradition and the institutionalisation of risk", en RANCI, C.; y PAVOLINI, E., Reforms in Long-term Care Policies in EU Countries, Nueva York, Springer.

RODRÍGUEZ CASTEDO, Á. (dir.) [2005]: Libro blanco de atención a las personas dependientes en situación de dependencia en España, Madrid, Imserso. [<http://www. imsersomayores.csic.es/documentos/ documentos/libroblancodependencia/mtaslibroblancodependencia-01.pdf〉].

RODRÍGUEZ RODRÍGUEZ, P. (2006): El sistema de servicios sociales español y las necesidades derivadas de la atención a la dependencia, serie Documentos de Trabajo, no 87, Madrid, Fundación Alternativas [rhttp:// www.falternativas.org/en/content/ download/5833/166791/version/1/ file/4cb8_04-07-06_27atencionDependencia. pdf>].

SANCHO CASTIELLO, M.; y RODRÍGUEZ RODRÍGUEZ, P. (2001): "Envejecimiento y protección social de la dependencia en España. Veinte años de historia y una mirada hacia el futuro", Intervención Psicosocial, vol. 10, nº 3, págs. 259-275 [khttp://www.copmadrid.org/ webcopm/publicaciones/social/76317.pdf)].

SOSVILLA RIVERO, S. (2010): Estimación del efecto del establecimiento del Sistema de Autonomía y Atención a la Dependencia sobre el empleo en España, Madrid, Mimeo. 